\title{
GENERALIZED SNELL'S LAW FOR WEIGHTED MINIMAL SURFACE IN HETEROGENEOUS MEDIA *
}

\author{
ZHILIN $\mathrm{LI}^{\dagger}$, XIAOBIAO LIN $^{\dagger}$, MONICA TORRES ${ }^{\ddagger}$, AND HONGKAI ZHAO
}

\begin{abstract}
The weighted minimal surface problem in heterogeneous media is studied in this paper. The solution to the weighted minimal surface problem is continuous but the derivatives have a jump across the interface where the medium property is discontinuous. The jump condition of the derivatives derived in this paper generalized the Snell's law in geometric optics to weighted minimal surfaces of co-dimension one in any dimensional space. A numerical method based on the gradient flow and the maximum principal preserving immersed interface method is developed to solve this nonlinear elliptic interface problem with jump conditions. Numerical computations are presented to verify both the analysis and the numerical algorithm.
\end{abstract}

1. Introduction. The minimal surface problem, that is, the problem of finding the surface of the least area among all surfaces having fixed boundary data, has been extensively studied. A recent workshop on minimal surfaces presented the latest research on minimal surface applications in chemistry and biology [5]. Many phenomena that occur in nature relate to this problem which has been a motivation for developing new mathematical theories and techniques to solve the problem analytically and numerically. Minimal surfaces were shown to be important in various chemical micro-structures and their corresponding phase transitions [5]. Computer graphics and image analysis use minimal surfaces frequently for boundary detection, and to construct surfaces that are visually appealing [2], [15]. Soap films and other membranes passing through a fixed boundary provide mechanical examples of minimal surfaces [14]. A related concept is the idea of capillary surfaces, which result from surface tension in liquids. These surfaces are closely related to minimal surfaces [6]. For a precise mathematical description of the minimal surface problem we refer, for example, to the classical treatises [7] and [16].

The minimal surface problem can be described in two different ways, using the parametric or the non-parametric formulation. In the non-parametric setting the candidate surfaces are graphs of functions, while in the parametric setting the surfaces are treated as boundaries of sets [7]. The former is usually seen in more physicallybased treatments of the problem, whereas the later provides an excellent framework for the mathematical analysis of minimal surfaces.

When the medium is homogeneous, the energy density at each point is constant, and therefore the surface energy is equivalent to the surface area. This is the standard minimal surface problem. In this paper, we consider the weighted minimal surface problem in a heterogeneous medium in which the energy density is piecewise smooth. For example this is the case for capillary interfaces in porous media or composite materials. In particular we derive a jump condition for the weighted minimal surface at the interface between two different media. The jump condition can be regarded as a generalized Snell's law which describes the "refraction" of minimal surfaces instead of light rays in geometric optics.

\footnotetext{
*Received January 4, 2003; accepted for publication October 24, 2003.

${ }^{\dagger}$ Center for Research in Scientific Computation \& Department of Mathematics, North Carolina State University, Raleigh, NC 27695, USA (zhilin@math.ncsu.edu, xblin@math.ncsu.edu).

${ }^{\ddagger}$ Department of Mathematics, Northwestern University, Evanston, IL 60208, USA (torres@math.northwestern.edu).

$\S$ Department of Mathematics, University of California, Irvine, 92697, USA (zhao@math.uci.edu).
} 
For simplicity, we restrict our discussion to a two dimensional surface in three dimensions. Our results extend to minimal surfaces of co-dimension one in higher dimensional spaces. Below we present the setup of the problem. Given a two dimensional domain $\Omega \subset R^{2}$, we want to find a function $u(x, y)$ which minimizes the weighted surface area of its graph with a prescribed Dirichlet boundary condition, i.e.,

$$
\begin{aligned}
& \min _{u} E(u)=\iint_{\Omega} c(x, y, u) \sqrt{1+|\nabla u(x, y)|^{2}} d x d y, \\
& \left.u(x, y)\right|_{\partial \Omega}=u_{0}(x, y)
\end{aligned}
$$

where $c(x, y, u)$ is a piecewise smooth function which has a finite jump across a surface defined by

$$
S=\{(x, y, z), \varphi(x, y, z)=0\}
$$

in three dimensional space, see Fig. 2.1 for an illustration. We assume that the surface is smooth and $\varphi(x, y, z)$ has up to second order continuous partial derivatives. In this paper we consider the case $c>0$. Mathematically, the case when $c=0$ has been studied in [17] using standard arguments of the calculus of variations. In [17], the minimal surfaces are treated as the boundary of sets of the finite perimeter (not graphs of functions) and the case with weight zero inside the medium and one outside is studied. In particular, minimal surfaces that always stay at a bounded distance (universal) from a given plane are constructed. For this degenerate case it can be proven [8] that the minimal surfaces enter the medium inside orthogonally which means, roughly speaking, that the intersection of the minimal surface and the boundary that separates two mediums locally looks like two perpendicular hyperplanes. Actually the orthogonality condition for the degenerate case $c=0$ can be formally derived through taking the limit of our generalized Snell's law in this paper, see [17].

In practice the computation of a weighted minimal surface in a piecewise smooth medium is quite challenging due to the nonlinearity of the Euler-Lagrange equation and the discontinuity of the coefficient across the interface. Furthermore, the solution to the variational form is not unique meaning there are multiple minima. A direct approach to minimize the variational form involves a three dimensional minimization algorithm and an evolution of two dimensional surface which is potentially expensive and complicated.

In this paper, we propose a numerical algorithm that reduces the problem to a two dimensional problem which can be solved by the existing immersed interface method (IIM) package for elliptic interface problems at each iteration. The intersection of the minimal surface and the interface is a free boundary problem that is coupled with the Euler-Lagrange equation through the generalized Snell's law. In our method, we represent the minimal surface as a graph function and formulate the generalized Snell's law as an interface condition for the graph function to reduce the computational cost. We linearize the equation and the jump condition by substituting the previous approximation in the nonlinear terms and use the maximum principle preserving scheme for parabolic interface problem $[1,12]$. A smoothing technique and a reinitialization process is used to regularize the problem numerically. Since the energy functional is not convex, there are local minima and hence the solution depends on the initial condition $u_{0}(x, y)$, see Fig. 4.4 for an example. 
The paper is organized as follows. In $\S 2$, we derive the Euler-Lagrange equation and the generalized Snell's law at the interface of two mediums. In $\S 3$, we present a general computational framework and outline the algorithm that is used to compute the weighted minimal surface. Some numerical examples are presented in $\S 4$. The paper is concluded in $\S 5$.

\section{Derivation of the Euler-Lagrangian equation and the jump condi-} tion.

2.1. The Euler-Lagrangian Equation. When $c \equiv 1$ in the entire domain, the minimal surface problem is commonly known as Plateau's Problem [4]. In general, there may be one, multiple, or no minimal surfaces satisfy the given boundary condition. The existence of a solution to the general case proved by Douglas (1931 [4]), and Radó (1933, [13]) could not exclude the possibility of singularities. In this paper, we only consider the case that the minimal surface is smooth except at the interface. Although our derivation of the Euler-Lagrangian equation is not new, we include it in details not only because that the weight $c$ is non-constant in our paper, but also it is needed in the second half of this section where the jump condition across the interface $S$ is considered.

Assume that $c(x, y, z)$ is a piecewise smooth function in $\mathbb{R}^{3}$ :

$$
c(x, y, z)= \begin{cases}c_{2}(x, y, z), & (x, y, z) \in B, \\ c_{1}(x, y, z), & (x, y, z) \in \mathbb{R}^{3}-B\end{cases}
$$

where $B=\{(x, y, z) \mid \varphi(x, y, z)<0\}$ is an open set in $\mathbb{R}^{3}$ with the smooth boundary $S=\{(x, y, z) \mid \varphi(x, y, z)=0\}$. Since $S$ is smooth, we can extend $c_{1}(x, y, z)$ and $c_{2}(x, y, z)$ smoothly across $S$ in a small neighborhood of $S$ from one side to the other for theoretical convenience.

Let $\Omega$ be an open set in $\mathbb{R}^{2}$ whose boundary $\partial \Omega$ is smooth. Assume that the cylindrical region $\Omega \times \mathbb{R}$ contains $B$. A function $u_{0}(x, y)$ on $\partial \Omega$ is specified. We look for a piecewise smooth function $u(x, y)$ in $\Omega$ that satisfies $\left.u(x, y)\right|_{\partial \Omega}=u_{0}(x, y)$ and minimizes $E(u)$ in (1.1). Assume that the graph of $u(x, y)$ intersects with the surface $S$ at a curve $\Gamma$, and the projection of $\Gamma$ to the $(x, y)$-plane is a smooth curve $\gamma$, which divides the domain $\Omega$ into two subsets $\Omega_{1}$ and $\Omega_{2}$. Assume also that the function $u(x, y)$ is smooth in each $\Omega_{j}, j=1,2$. Across $\gamma$, the function $u(x, y)$ is continuous but $\left(u_{x}, u_{y}\right)$ usually has a finite jump.

$$
\begin{gathered}
\text { Let } \gamma(s)=\{(x(s), y(s)) \mid a \leq s \leq b, x(a)=x(b), y(a)=y(b)\} \text {, and } \\
F\left(x, y, z, z_{x}, z_{y}\right)=c(x, y, z) \sqrt{1+z_{x}^{2}+z_{y}^{2}} .
\end{gathered}
$$

With $z=u(x, y)$, the variational equation of $E(u)$ is

$$
\delta E(u)=\iint_{\Omega}\left(F_{z} \delta z+F_{z_{x}} \delta z_{x}+F_{z_{y}} \delta z_{y}\right) d x d y=0,
$$

where $\delta z$ is a variation of $z$ and satisfies $\left.\delta z\right|_{\partial \Omega}=0$.

TheOREM 2.1. If $u(x, y)$ is $C^{2}$ in $\Omega_{1} \cup \Omega_{2}$ and a local minimum of (1.1), then it satisfies the following Euler-Lagrangian equation in the open set $\Omega_{1} \cup \Omega_{2}$ :

$$
\frac{\partial}{\partial x}\left(\frac{c u_{x}}{\sqrt{1+u_{x}^{2}+u_{y}^{2}}}\right)+\frac{\partial}{\partial y}\left(\frac{c u_{y}}{\sqrt{1+u_{x}^{2}+u_{y}^{2}}}\right)=c_{z}(x, y, u) \sqrt{1+u_{x}^{2}+u_{y}^{2}}
$$




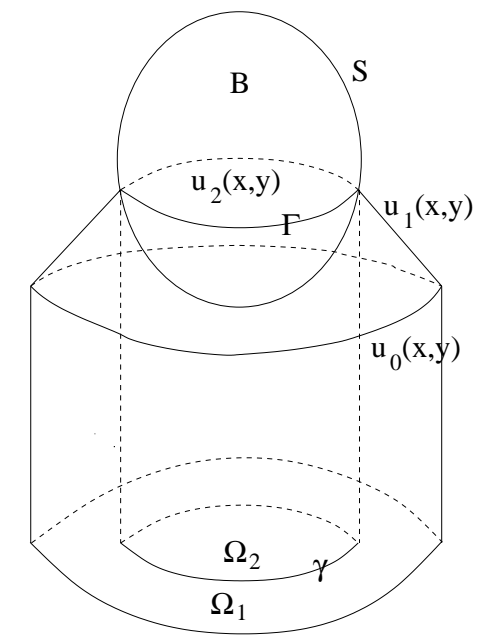

FIG. 2.1. A diagram of the weighted minimal surface problem. The solution $u(x, y)$ is piecewise smooth but has a finite jump in the partial derivatives across $\gamma$.

Proof. Assume that the graph of $u(x, y)$ passes through $\Gamma$, i.e., $\left.\delta z\right|_{\gamma}=0$. If $u$ is a local minimizer, we must have $\delta E=0$ for such $\delta z$. Using the Green's formula, we have

$$
\begin{aligned}
\delta E & =\iint_{\Omega_{1}}+\iint_{\Omega_{2}}\left(F_{z} \delta z+F_{\delta z_{x}} \delta z_{x}+F_{\delta z_{y}} \delta z_{y}\right) d x d y \\
& =\iint_{\Omega_{1}}+\iint_{\Omega_{2}}\left(F_{z}-\frac{\partial}{\partial x} F_{z_{x}}-\frac{\partial}{\partial y} F_{z_{y}}\right) \delta z d x d y \\
& =0 .
\end{aligned}
$$

Since $\delta z$ is arbitrary in $\Omega_{1} \cup \Omega_{2}$, we must have

$$
F_{z}-\frac{\partial}{\partial x} F_{z_{x}}-\frac{\partial}{\partial y} F_{z_{y}}=0, \quad \Omega_{1} \cup \Omega_{2} .
$$

The Euler-Lagrangian equation (2.2) follows by plugging $F$ into (2.3).

We will assume that $z=u(x, y)$ satisfies the Euler-Lagrange equation (2.3) in the rest of the paper.

\subsection{The jump condition across the interface. Let}

$$
\Gamma_{0}=\left\{\left(x_{0}(s), y_{0}(s), z_{0}(s)\right) \mid z_{0}(s)=u\left(x_{0}(s), y_{0}(s)\right)\right\}
$$

be the intersection of the minimal surface and $S$. Let $\Gamma=\Gamma_{0}+\delta \Gamma$ be a closed curve near $\Gamma_{0}$. For every small perturbation $\delta \Gamma$, we assume that there is unique solution $u+\delta u$ to the Euler-Lagrangian equation in $\Omega_{1}$ and $\Omega_{2}$ that passes through $\Gamma$ and satisfies the boundary condition at $\partial \Omega$. In this subsection we study the variation $\delta E(u)$ with respect to $\delta \Gamma$ and derive the jump condition for the solution $u$ across $\gamma$. The result is summarized in the following theorem. 
ThEOREM 2.2. Assume that, in a neighborhood of $\Gamma$, the surface $S=$ $\{\varphi(x, y, z)=0\}$ can be expressed as $z=\phi(x, y)$. Let

$$
\mathbf{p}_{j}=\frac{\left(-u_{x},-u_{y}, 1\right)}{\sqrt{1+u_{x}^{2}+u_{y}^{2}}}, \quad \mathbf{q}=\frac{\left(-\phi_{x},-\phi_{y}, 1\right)}{\sqrt{1+\phi_{x}^{2}+\phi_{y}^{2}}}, \quad j=1,2
$$

be the unit normal directions of the surface $u(x, y)$ in $\Omega_{j}$, and the unit normal direction of $S$ respectively, all pointing to the positive direction in $z$ direction. Then if $u(x, y)$ is the solution of the weighted minimal surface problem, the jump of the derivatives of $u(x, y)$ across the surface $S$ must satisfy the following generalized Snell's Law:

$$
\left.c_{1} \mathbf{p}_{1} \cdot \mathbf{q}\right|_{\Gamma}=\left.c_{2} \mathbf{p}_{2} \cdot \mathbf{q}\right|_{\Gamma},
$$

where $\Gamma$ is the intersection of the minimal surface and the interface $S$.

Proof. To derive the jump condition for a minimizer $u(x, y)$ across $\gamma$, we shall compute the variation of $E(u)$ due to the variation of $\Gamma$.

The variation of $\Gamma$ naturally decomposes into the vertical and horizontal directions. We first assume the variation of $\Gamma$ is vertical, i.e.

$$
\delta \Gamma=\{(0,0, \delta z(s))\}
$$

Let $\delta E=\delta E_{1}+\delta E_{2}$ where $\delta E_{j}$ is the variation in $\Omega_{j}, j=1,2$,

$$
\delta E_{j}=\iint_{\Omega_{j}}\left(F_{z} \delta z+F_{z_{x}} \delta z_{x}+F_{z_{y}} \delta z_{y}\right) d x d y
$$

Using the Euler-Lagrange equation in $\Omega_{j}$ and the Green's formula, we have the variational equation due to the vertical variation of $\Gamma$ :

$$
\delta E_{j}=-\oint_{\gamma} \delta z(s)\left(F_{z_{x}}, F_{z_{y}}\right) \cdot \mathbf{n}_{\mathbf{j}}(s) d s, \quad j=1,2,
$$

where $\mathbf{n}_{j}(s)$ is the inward unit normal vector of $\Omega_{j}$ at $\gamma(s)$.

Next, we consider the variation of $E_{j}$ due to the variation in $\Gamma$ on the surface of the graph of $u(x, y)$. To compute $\delta E_{1}$, we will move $\gamma$ into $\Omega_{1}$, and to compute $\delta E_{2}$, we will move $\gamma$ into $\Omega_{2}$. Each $\delta E_{j}$ will be treated as a linear functional of $\delta \Gamma$ and will be obtained from a one-sided differentiation of $E_{j}$ with respect to $\delta \Gamma$ in function spaces.

In each $\Omega_{j}$, in a small neighborhood of $\gamma$, we introduce a local coordinate system

$$
x=x(s, n), y=y(s, n),
$$

where $s$ the the arc length along $\gamma$, and $n$ is the distance to $\gamma(s)$ along the inward normal direction of $\Omega_{j}$ at $\gamma(s)$. Assume that $n$ is sufficiently small, then the change of coordinates $(x, y) \rightarrow(n, s)$ is nonsingular and $d x d y \approx d s d n$. In fact, $d x d y=$ $\left|\frac{\partial(x, y)}{\partial(s, n)}\right| d s d n$ and the determinant is unity at $n=0$. Therefore, we have

$$
\left|\frac{\partial(x, y)}{\partial(s, n)}\right|=1+O(\delta n(s))
$$



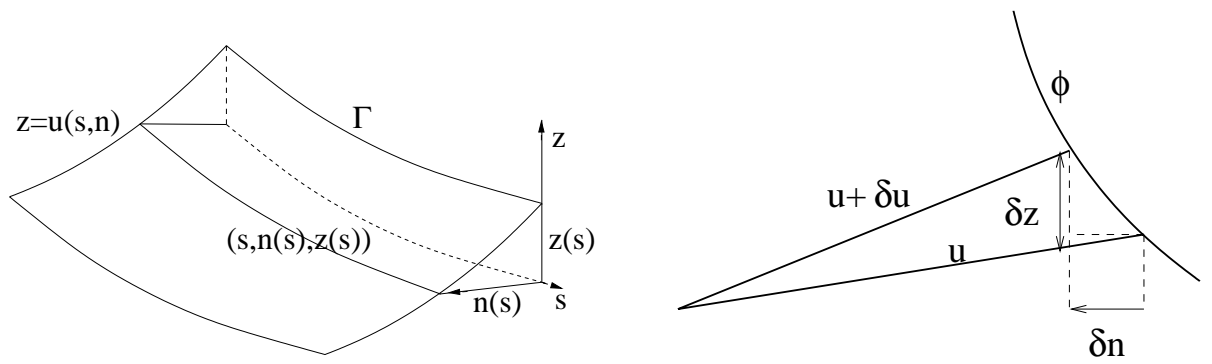

FIG. 2.2. The variation of $\Gamma$ and $u$ near the interface $S$.

Let $\delta \Gamma(s)=(x(s, \delta n(s)), y(s, \delta n(s), \delta z(s))$, where $\delta n(s) \geq 0$ and $\delta z(s)$ is chosen so that $\Gamma+\delta \Gamma$ is on the graph of $u(x, y)$. Denoted the region bounded by $\gamma$ and $\gamma+\delta \gamma$ as $\delta \Omega_{j}$. Tracking the lowest order terms, we have

$$
\begin{aligned}
\delta E_{j} & =-\iint_{\delta \Omega_{j}} F d x d y \\
& =-\iint_{\delta \Omega_{j}} F\left|\frac{\partial(x, y)}{\partial(x, n)}\right| d s d n \\
& =-\oint_{\gamma} \int_{0}^{\delta n(s)}(1+O(\delta n(s))) F d n d s \\
& =-\oint_{\gamma} F(s) \delta n(s) d s+O\left((\delta n(s))^{2}\right) .
\end{aligned}
$$

Dropping the higher order terms, we have

$$
\delta E_{j}=-\oint_{\gamma} F(s) \delta n(s) d s .
$$

Note that

$$
\delta E_{j}=\frac{\partial E_{j}}{\partial n} \delta n+\frac{\partial E_{j}}{\partial z} \delta z .
$$

Here $\frac{\partial E_{j}}{\partial n}$ and $\frac{\partial E_{j}}{\partial z}$ are linear functionals on $\delta n$ and $\delta z$.

From (2.5), we have

$$
\frac{\partial E_{j}}{\partial z} \delta z=-\oint_{\gamma}\left(\left(F_{z_{x}}, F_{z_{y}}\right) \cdot \mathbf{n}\right) \delta z(s) d s .
$$

Combining this with (2.6) and (2.7), we have

$$
\frac{\partial E_{j}}{\partial n} \delta n=-\oint_{\gamma} F(s) \delta n(s) d s+\oint_{\gamma}\left(\left(F_{z_{x}}, F_{z_{y}}\right) \cdot \mathbf{n}\right) \delta z(s) d s .
$$

Since we assume that $\Gamma$ is on the surface of $u(x, y)$, see Fig. 2.2(a), there is a relation between the vertical and horizontal variation:

$$
\delta z(s)=\frac{\partial u}{\partial n} \delta n(s) .
$$


Thus we have found the variational equation due to the horizontal variation of $\Gamma$ :

$$
\frac{\partial E_{j}}{\partial n} \delta n=\oint_{\gamma}\left(-F(s)+\left(F_{z_{x}}, F_{z_{y}}\right) \cdot \mathbf{n} \frac{\partial u}{\partial n}\right) \delta n(s) d s .
$$

To keep $\Gamma+\delta \Gamma$ on the surface $S$ where $c$ has a jump discontinuity, we assume that $\delta z(s)=\frac{\partial \phi}{\partial n} \delta n(s)$ in $(2.5)$. So the total change in $z$ direction due to the perturbation $\delta n(s)$ is $\left(\frac{\partial \phi}{\partial n}-\frac{\partial u}{\partial n}\right) \delta n(s)$, see Fig. 2.2(b). Thus the total variation for $\Gamma$ on $S$ is

$$
\delta E_{j}=\oint_{\gamma}\left(-F(s)+\left(F_{z_{x}}, F_{z_{y}}\right) \cdot \mathbf{n}\left(\frac{\partial u}{\partial n}-\frac{\partial \phi}{\partial n}\right)\right) \delta n(s) d s, j=1,2 .
$$

Define

$$
\begin{aligned}
\tilde{F}\left(s, n, z, z_{s}, z_{n}\right) & :=F\left(x, y, z, z_{x}, z_{y}\right) \\
& =F\left(x(s, n), y(s, n), z(s, n), \frac{\partial s}{\partial x} z_{s}+\frac{\partial n}{\partial x} z_{n}, \frac{\partial s}{\partial y} z_{s}+\frac{\partial n}{\partial y} z_{n}\right) .
\end{aligned}
$$

Note that $\mathbf{n}=\left(\frac{\partial n}{\partial x}, \frac{\partial n}{\partial y}\right)$. Thus we have

$$
\tilde{F}_{z_{n}}=F_{z_{x}} \frac{\partial n}{\partial x}+F_{z_{y}} \frac{\partial n}{\partial y}=\left(F_{z_{x}}, F_{z_{y}}\right) \cdot \mathbf{n} .
$$

Denoting $\tilde{F}$ by $F$ if no confusion should arise, we have:

$$
\delta E_{j}=-\oint_{\gamma}\left(F(s)+F_{z_{n}}\left(\frac{\partial \phi}{\partial n}-\frac{\partial u}{\partial n}\right)\right) \delta n(s) d s, j=1,2 .
$$

More generally, let $\delta n(s)$ be an arbitrarily function of $s$, not necessarily nonnegative as in the previous paragraphs. Since in $\Omega_{1}$ and $\Omega_{2}$, the inward normal directions are opposite to each other, if $\delta n(s)=\psi(s)$ in $\Omega_{1}$, then $\delta n(s)=-\psi(s)$ in $\Omega_{2}$ where $\psi(s)$ is an arbitrarily smooth function of $s$. The total variation in $\Omega_{1} \cup \Omega_{2}$ must add up to zero, therefore, we have the jump condition across the surface $S$ :

$$
\left.\left(F(s)+F_{z_{n}}\left(\frac{\partial \phi}{\partial n}-\frac{\partial u}{\partial n}\right)\right)\right|_{j=1}=\left.\left(F(s)+F_{z_{n}}\left(\frac{\partial \phi}{\partial n}-\frac{\partial u}{\partial n}\right)\right)\right|_{j=2} .
$$

We now apply (2.11) to the minimal surface problem with $F$ given in (2.1). Using (2.9), we have

$$
\begin{aligned}
F_{z_{n}} & =\left(\frac{c u_{x}}{\sqrt{1+u_{x}^{2}+u_{y}^{2}}}, \frac{c u_{y}}{\sqrt{1+u_{x}^{2}+u_{y}^{2}}}\right) \cdot \mathbf{n} \\
& =\frac{c u_{n}}{\sqrt{1+u_{x}^{2}+u_{y}^{2}}} .
\end{aligned}
$$

Note that along the line $\Gamma$ where $u(s, n)$ and $\phi(s, n)$ meet, $u(s, 0)=\phi(s, 0), u_{s}=$ $\phi_{s}$ and $1+u_{x}^{2}+u_{y}^{2}=1+u_{s}^{2}+u_{n}^{2}$. From (2.12), on the line $\Gamma$, for $j=1,2$, we have,

$$
\begin{aligned}
F(s)+F_{z_{n}}\left(\frac{\partial \phi}{\partial n}-\frac{\partial u}{\partial n}\right) & =\frac{c(x, y, u)\left(1+u_{s} u_{s}+u_{n} \phi_{n}\right)}{\sqrt{1+u_{s}^{2}+u_{n}^{2}}} \\
& =\frac{c(x, y, u)\left(1+u_{s} \phi_{s}+u_{n} \phi_{n}\right)}{\sqrt{1+u_{s}^{2}+u_{n}^{2}}}
\end{aligned}
$$


If we multiply both sides of $(2.11)$ by $1 / \sqrt{1+\phi_{s}^{2}+\phi_{n}^{2}}$, which is continuous across the interface, and use $u_{x} \phi_{x}+u_{y} \phi_{y}=u_{s} \phi_{s}+u_{n} \phi_{n}$, we have finally arrived at $(2.4)$.

REMARKS.

1. If the interface $S$ is expressed in terms of the zero level surface $\varphi(x, y, z)=0$, then the jump condition is equivalent to

$$
\left[\frac{c\left(u_{x} \varphi_{x}+u_{y} \varphi_{y}\right)}{|\nabla \varphi| \sqrt{1+|\nabla u|^{2}}}\right]=\left[\frac{c \varphi_{z}}{|\nabla \varphi| \sqrt{1+|\nabla u|^{2}}}\right]
$$

where $\left(\varphi_{x}, \varphi_{y}, \varphi_{z}\right) /|\nabla \varphi|$ is the unit normal direction of the surface $S$.

2. The jump condition (2.4) is a local condition and can be written in a coordinate free form. Define the dihedral angle $\theta$ between two surfaces to be the angle between their normals. Then the generalized Snell's law (2.4) can be expressed as

$$
c_{1} \cos \left(\theta_{1}\right)=c_{2} \cos \left(\theta_{2}\right),
$$

where $\theta_{j}$ is the dihedral angle between the interface $S$ and the surface $u_{j}(x, y)$. Our derivations and the generalized Snell's law extend to weighted minimal surfaces of co-dimension one in any number of dimensions.

3. A numerical method. Numerical computation of weighted minimal surfaces in heterogeneous media in three dimensions is not easy. First of all the Euler-Lagrange equation is nonlinear. Moreover, we need to incorporate the generalized Snell's law across a free boundary, the intersection of the minimal surface and the interface between two different media. The main difficult is that several tasks, tracking the free boundary, enforcing the jump condition across the free boundary, and solving the nonlinear partial differential equation in separated regions, have to be handled simultaneously and consistently.

In this paper, we propose an iterative approach based on the gradient flow. We represent the minimal surface as a graph and reformulate the the generalized Snell's law as a two dimensional interface condition for the graph function so that we only need to solve the Euler Lagrange equation in two space dimensions. We take advantage of the maximum principle preserving scheme for linear elliptic interface problems [12].

In this section, we assume that $c$ is a piecewise constant with a finite jump across the interface $S$. We would like to compute the solution to the Euler-Lagrange equation

$$
\frac{\partial}{\partial x}\left(\frac{c u_{x}}{\sqrt{1+|\nabla u|^{2}}}\right)+\frac{\partial}{\partial y}\left(\frac{c u_{y}}{\sqrt{1+|\nabla u|^{2}}}\right)=0, \quad(x, y) \in \Omega_{1} \cup \Omega_{2}-\gamma
$$

with the jump condition (2.14) directly. Due to the difficulties mentioned earlier, a direct solver to the elliptic non-linear interface problem is very challenging. Moreover, the solution may not be unique, and may be very sensitive to the boundary conditions and the geometric configuration of the interface, see Section 4. To prevent the approximate solution from jumping from one solution to another, we solve the following gradient flow, a nonlinear parabolic equation, to steady state

$$
\begin{aligned}
u_{t} & =\frac{\partial}{\partial x}\left(\frac{c u_{x}}{\sqrt{1+|\nabla u|^{2}}}\right)+\frac{\partial}{\partial y}\left(\frac{c u_{y}}{\sqrt{1+|\nabla u|^{2}}}\right) \\
\left.u(x, y, t)\right|_{\partial \Omega} & =u_{0}(x, y),
\end{aligned}
$$


where $u_{0}(x, y)$ is the boundary condition. An initial guess that satisfies the boundary condition has to be chosen and the time step can be adjusted depending on the geometry of the problem. Note that, we need to use the parabolic equation (3.2) only when the numerical solution shows signs of branching, see Fig. 4.3 for an example.

3.1. The outline of the algorithm. We express the boundary $S$ between the two different mediums as the zero level set of a function $\varphi(x, y, z)$ :

$$
\varphi(x, y, z) \begin{cases}<0, & \text { if }(x, y, z) S \\ =0, & \text { if }(x, y) \text { is on } S \\ >0, & \text { if }(x, y) \text { is outside of } S\end{cases}
$$

The actual computation is done on a two dimensional domain $[a, b] \times[c, d]$. We use a uniform Cartesian grid

$$
x_{i}=a+i h_{x}, \quad i=0,1, \cdots, M ; \quad y_{j}=c+j h_{y}, \quad j=0,1, \cdots, N,
$$

where $h_{x}=\frac{b-a}{M}$ and $h_{y}=\frac{b-a}{N}$. We need to carefully choose an initial guess $u^{0}(x, y)$ that satisfies the boundary condition in order to get the intended solution. We use the substitution method, see [9] for example, to find an approximate solution to the weighted minimal surface problem. The main procedure from $u^{k}(\mathbf{x})$ to get $u^{k+1}(\mathbf{x})$ is outlined below:

- Step 1: Regularize the numerical solution by the following modified Helmholtz equation ${ }^{1}$

$$
u^{*}-\Delta t_{1} \Delta u^{*}=u^{k}
$$

where $\Delta t_{1}$ is a regularization factor and is of order $h^{2}$, and the Laplacian operator is discretized in the standard way:

$$
\Delta_{h} u_{i j}^{*}=\frac{u_{i-1, j}^{*}-2 u_{i j}^{*}+u_{i+1, j}^{*}}{\left(h_{x}\right)^{2}}+\frac{u_{i, j-1}^{*}-2 u_{i j}^{*}+u_{i, j+1}^{*}}{\left(h_{y}\right)^{2}} .
$$

The purpose of this step is to smooth the intermediate approximation $u^{k}(x, y)$ without changing it too much. This step is introduced because the intermediate solution to the interface problem usually has non-smooth second order derivatives, and this may affect the convergence of our method. We use the fast solver from the Fishpack which is available through the Netlib to solve the above equation. We gradually decrease $\Delta t_{1}$ as $\left\{u^{k}\right\}$ appears to satisfies our convergence criterion.

- Step 2: Set $\varphi_{2}^{k}\left(x_{i}, y_{j}\right)=\varphi\left(x_{i}, y_{j}, u_{i j}^{*}\right)$. If $\varphi_{2}^{k}(x, y)=0$ is non-empty, that is

$$
\max _{i j}\left\{\varphi_{2}^{k}\left(x_{i}, y_{j}\right)\right\} \min _{i j}\left\{\varphi_{2}^{k}\left(x_{i}, y_{j}\right)\right\}<0,
$$

we use a re-initialization scheme so that $\varphi_{2}^{k}(x, y)$ is a good approximation to the signed distance function to the set $\varphi_{2}^{k}(x, y)=0$, and has up to second order partial derivatives in the neighborhood of its zero level set. This is a

\footnotetext{
${ }^{1}$ This step is used for the purpose of stabilizing the iterative procedure and accelerating the convergence especially when subtle situation occurs (see the example in Figure 4.3).
} 
standard technique in the level set method. The re-initialization is essential to the algorithm because $\varphi\left(x, y, u^{*}(x, y)\right)$ is usually a non-differentiable function across the curve $\varphi\left(x, y, u^{*}(x, y)\right)=0$.

- Step 3: Update $u^{k+1}$ from

$$
\begin{aligned}
& u^{k+1}=u^{*}+\Delta t_{2}\left\{\frac{\partial}{\partial x}\left(\frac{c u_{x}^{k+1}}{\sqrt{1+\left|\nabla u^{*}\right|^{2}}}\right)+\frac{\partial}{\partial y}\left(\frac{c u_{y}^{k+1}}{\sqrt{1+\left|\nabla u^{*}\right|^{2}}}\right)\right\} \\
& \left.u^{k+1}(x, y)\right|_{\partial \Omega}=u_{0}(x, y)
\end{aligned}
$$

where $\Delta t_{2}$ is the time step. If the zero level set $\varphi_{2}^{k}(x, y)=0$ is non-empty, we reformulate the jump condition $(2.14)$ across $\varphi_{2}^{k}(x, y)=0$ as

$$
\begin{gathered}
{\left[u^{k+1}\right]=0} \\
{\left[\frac{c u_{n}^{k+1}}{\sqrt{1+\left|\nabla u^{*}\right|^{2}}}\right]=\left[\frac{c}{\sqrt{1+\left|\nabla u^{*}\right|^{2}}} \frac{\varphi_{z}}{|\nabla \varphi|}\right]} \\
+\left[\frac{c}{\sqrt{1+\left|\nabla u^{*}\right|^{2}}}\left(\left(n_{x}^{k}-\frac{\varphi_{x}}{|\nabla \varphi|}\right) u_{x}^{*}+\left(n_{y}^{k}-\frac{\varphi_{y}}{|\nabla \varphi|}\right) u_{y}^{*}\right)\right],
\end{gathered}
$$

where $\mathbf{n}^{k}=\left(n_{x}^{k}, n_{y}^{k}\right)$ is the unit normal direction of the plane curve $\varphi_{2}^{k}(x, y)=$ $\varphi\left(x, y, u^{*}(x, y)\right)=0$ pointing outward. The gradient $\nabla \varphi$ is computed at $\left(x, y, u^{*}(x, y)\right)$. It is obvious that if $u^{k}$ converges, the modified jump condition (3.8) converges to the true jump condition (2.14). Since we are only interested in the steady state solution of (3.2), the accuracy in time variable is unimportant. We use the maximum principal preserving scheme to discretize the elliptic part, that is the right hand side of equation $(3.2)$, see $[12,1]$. The discrete implicit finite difference equation is solved using the multigrid solver [3]. Note that we are only interested in the steady state solution of the parabolic problem and $\Delta t_{2}$ is an auxilliary parameter. Thus we can take larger $\Delta t_{2}$ within the stability region and gradually decrease $\Delta t_{2}$ as $\left\{u^{k}\right\}$ appears to satisfies our convergence criterion.

Note that we have re-written the jump condition to fit perfectly into our numerical scheme for the elliptic interface problems described in [12]. The crucial part in the algorithm is to compute the gradient $\nabla u^{*}$ at staggered grid points $\left(x_{i} \pm h_{x} / 2, y_{j}\right)$ and $\left(x_{i}, y_{j} \pm h_{y} / 2\right)$, and certain points on the interface $\varphi_{2}^{k}(x, y)=0$. The algorithm is described in the following subsection. The main methodology is the least square interpolation techniques, see $[9,11]$.

3.2. Interpolation scheme for $\nabla u^{*}$. In each iteration, we need to compute $\nabla u^{*}$ at grid points for the differential equation (3.7) and at some points on the intermediate interface to get the jump condition. Note that, $u^{*}$ is non-smooth, a direct finite difference scheme is inaccurate. We distinguish two different cases. For simplicity of notation, we simply use $u$ instead of $u^{*}$.

Case I: For a regular grid point $\left(x_{i}, y_{j}\right)$ which is away from the interface, we use the formula:

$2|\nabla u|_{i j}^{2}=\left(\frac{u_{i+1, j}-u_{i j}}{\Delta x}\right)^{2}+\left(\frac{u_{i j}-u_{i-1, j}}{\Delta x}\right)^{2}+\left(\frac{u_{i, j+1}-u_{i j}}{\Delta y}\right)^{2}+\left(\frac{u_{i j}-u_{i, j-1}}{\Delta y}\right)^{2}$. 
Such approximation is second order accurate and usually is slightly better than the central finite difference if the gradient is large.

Case II: For a point $\mathbf{X}^{*}=\left(x^{*}, y^{*}\right)$ near or on the intermediate interface, which can be an irregular grid point $\left(x_{i}, y_{j}\right)$ as well, we wish to get a one-sided second order approximation of $u_{x}$ and $u_{y}$ at $\left(x^{*}, y^{*}\right)$. Denote that $\Omega^{-}=\left\{(x, y), \varphi_{2}(x, y) \leq 0\right\}$ and assume that $\varphi_{2}\left(x^{*}, y^{*}\right) \leq 0$. We use the least squares interpolation scheme to get an approximation for $u_{x}$ and $u_{y}$ from a particular side, for example, from $\Omega^{-}$side,

$$
u_{x}^{-}\left(\mathbf{X}^{*}\right)=\sum_{\mathbf{x}_{i j} \in \Omega^{-},\left|\mathbf{x}_{i j}-\mathbf{X}^{*}\right| \leq R_{\epsilon}} \gamma_{i j} u_{i, j},
$$

where $\mathbf{x}_{i j}=\left(x_{i}, y_{j}\right), R_{\epsilon}$ is a pre-chosen parameter between $4.1 h \sim 6.1 h$. The summation is taken over the neighboring grid points of $\left(x^{*}, y^{*}\right)$. To get second order interpolation, $R_{\epsilon}$ is chosen in such a way that at least six grid points from $\Omega^{-}$side are enclosed. If there are more than six grid points are enclosed, we take $n_{s}$ points for the interpolation, where $6 \leq n_{s} \leq 12$. In other words, we take the first $n_{s}$ closest points to $\mathbf{X}^{*}$ from the set of $\left|\mathbf{x}_{i j}-\mathbf{X}^{*}\right| \leq R_{\epsilon}$. Note that the coefficients $\gamma_{i j}$ depends on $\mathbf{X}^{*}$, but for simplicity, we have omitted the dependency. The same procedure is applied to compute other one-sided derivatives.

4. Numerical examples. In this section, we will use the notation $c^{-}(\varphi(x, y) \leq$ $0)$ and $c^{+}(\varphi(x, y)>0)$ instead of $c_{1}$ and $c_{2}$. We first present an example in which the interface is a cylindrical surface. In this case, we have a fixed projected interface in the $x-y$ plane and a homogeneous jump condition across the interface because $\varphi_{z} \equiv 0$, $n_{x}^{k}=\varphi_{x} /|\nabla \varphi|$, and $n_{y}^{k}=\varphi_{y} /|\nabla \varphi|$. There is a unique solution and the substitution method converges, see [9] for the details. We omit the step 1 and take $\Delta t_{2}=\infty$ meaning that we solve the elliptic equation in (3.7) directly. Given an initial guess $u^{0}(x, y)$, the substitution method simply is

$$
\begin{aligned}
& \left(\frac{\partial}{\partial x} \frac{c u_{x}^{k+1}}{\sqrt{1+\left|\nabla u^{k}\right|^{2}}}\right)+\frac{\partial}{\partial y}\left(\frac{c u_{y}^{k+1}}{\sqrt{1+\left|\nabla u^{k}\right|^{2}}}\right)=0,\left.\quad u^{k+1}(x, y)\right|_{\partial \Omega}=u_{0}(x, y) \\
& {\left[u^{k+1}\right]=0, \quad\left[\frac{c u_{n}^{k+1}}{\sqrt{1+\left|\nabla u^{k}\right|^{2}}}\right]=0}
\end{aligned}
$$

In Fig. 4.1 (a)-(b), we show the mesh and contour plots of the minimal surface with $c^{-}=0.01$ and $c^{+}=1$ given the initial guess

$$
u^{0}(x, y)=-\frac{1}{2}+\frac{x+1}{2}, \quad \Omega=[-1,1] \times[-1,1]
$$

The Dirichlet boundary condition is $\left.u(x, y)\right|_{\partial \Omega}=\left.u^{0}(x, y)\right|_{\partial \Omega}$. The interface is the cylinder defined by $\varphi(x, y, z)=\frac{x^{2}}{0.6^{2}}+\frac{y^{2}}{0.5^{2}}-1$. If the two mediums are the same, that is $c^{+}=c^{-}$, then $u^{0}(x, y)$ is the unique solution. This has been confirmed by our code to check the consistency. When $c^{+}$is bigger than $c^{-}$which is the case in Fig. 4.1 (a)-(b), the minimal surface would try to avoid to pass outside the cylinder as much as possible to decrease the contribution from outside. The large area inside the cylinder was balanced by the smaller weight $c^{-}$.

In Fig. 4.1 (c)-(d), we show the results of an opposite case with $c^{-}=1$ and $c^{+}=0.01$. The cylinder is more complicated and is given by $r=0.5+0.2 \sin (4 \theta)$, $0 \leq \theta \leq 2 \pi$ in polar coordinates, where $r=\sqrt{x^{2}+y^{2}}$. Now the weighted minimal 
surface tries to avoid inside as much as possible that is the case in Fig. 4.1 (c). In Fig. 4.1 (b) and (d), the dotted line is the intersection of the cylinder and the minimal surface. The number of iterations of the substitution method is typically between $10 \sim 20$ iterations with the tolerance $10^{-6}$.

(a)

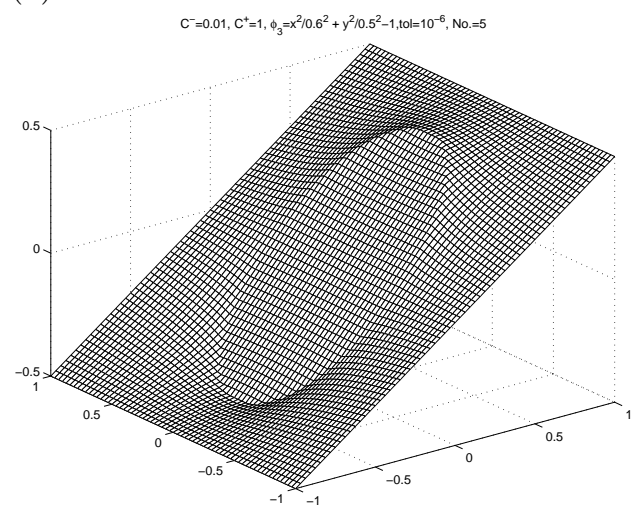

(c)

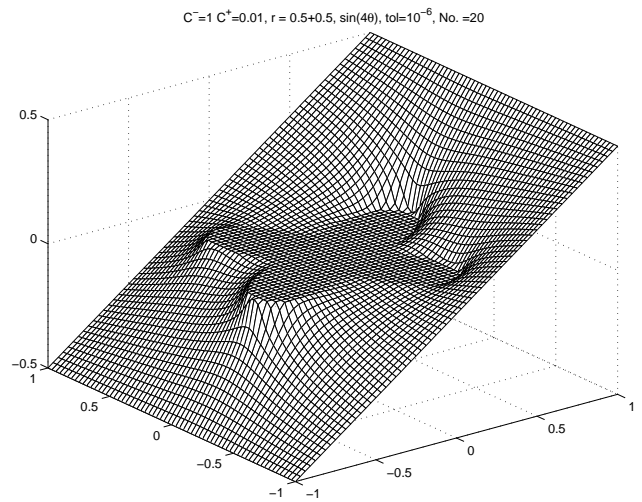

(b)

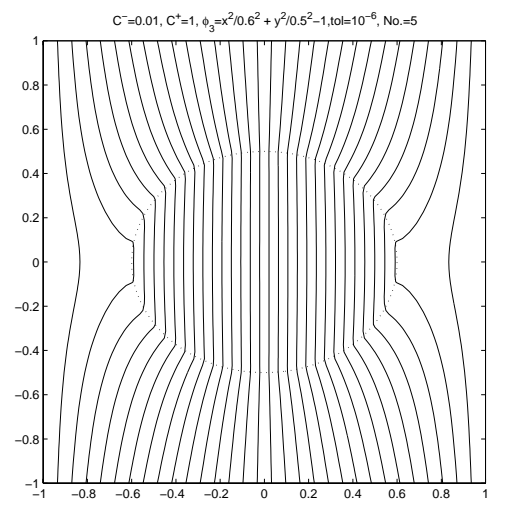

(d)

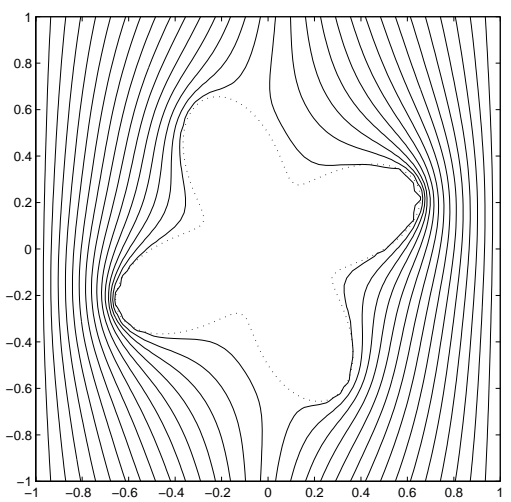

FIG. 4.1. Computed results with different coefficients $c$ and different cylinders. (a)-(b): Mesh and contour plots of the computed minimal surface $z=u(x, y)$ with $c^{-}=0.01, c^{+}=1$. The interface that separates the two mediums is the elliptic cylinder $\frac{x^{2}}{0.6^{2}}+\frac{y^{2}}{0.52}=1$. In (b), the dotted line is the intersection of $\varphi(x, y, z)=0$ and $\varphi(x, y, u(x, y))=0$. (c) $-(d)$ : Mesh and contour plots of the computed minimal surface $z=u(x, y)$ with $c^{-}=1, c^{+}=0.01$. The interface that separates the two mediums is the cylinder $r=0.5+0.2 \sin (4 \theta)$ in polar coordinates.

Now we present a numerical example in which the interface is a closed surface in three dimensional space. We take the interface that separates the two mediums as a sphere $x^{2}+y^{2}+z^{2}=0.75^{2}$. This is a much tougher example than the cylinder case because the intersection of the minimal surface and the interface becomes a free boundary which changes with the solution in each iteration, so does the the projected curve $\varphi(x, y, u(x, y))=0$. The final solution depends on both the boundary condition and the initial guess. The weighted minimal surface may or may not pass through or touch the interface that separates the two media. In order to avoid rapid changes in the solution, we choose $\Delta t_{1}=h^{2} / 4$ and $\Delta t_{2}=h^{2} / 4$.

In Fig. 4.2 (a) and (b), we show the computed weighted minimal surface with $c^{-}=2$ and $c^{+}=1$ in (a), and $c^{-}=1$ and $c^{+}=5$ in (b). The Dirichlet boundary 
condition is taken from the function $u(x, y)=-0.4+\frac{x+1}{2}$. The difference in $c$ is small and the numerical method can always give convergent results independent of the initial guess.

(a)

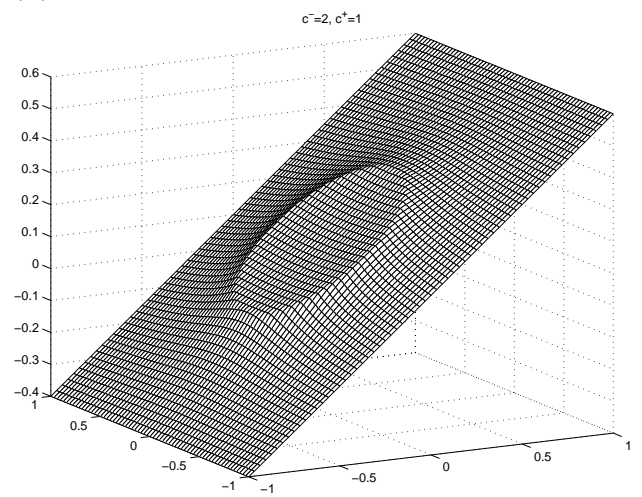

(b)

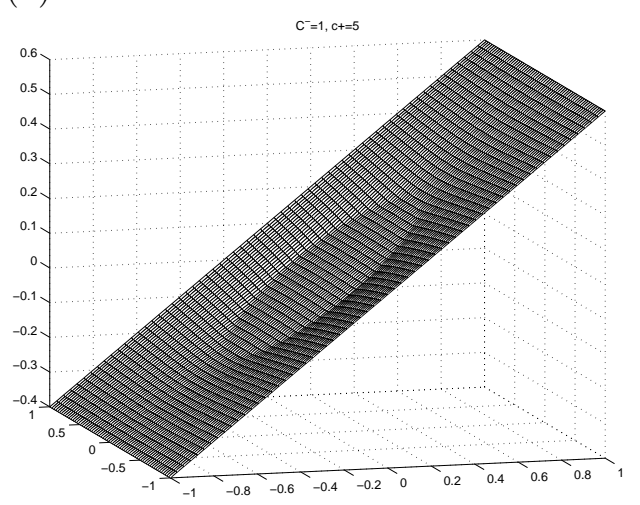

FIG. 4.2. Computed results with different coefficients $c$ with a sphere that separates the two mediums. The numerical results are independent of the initial guess. (a) $c^{-}=2, c^{+}=1$. (b) $c^{-}=1, c^{+}=5$.

TABLE 4.1

The grid refinement analysis against the solution from the finest mesh, $M=N=512$, for the case $c^{-}=2$ and $c^{+}=1$, see Fig. 4.2 (a). The results show nearly second order convergence.

\begin{tabular}{||c||c|c||}
\hline \hline$M \times N$ & $\left\|E_{N}\right\|_{\infty}$ & ratio \\
\hline $32 \times 32$ & $1.3458 \times 10^{-1}$ & \\
\hline $64 \times 64$ & $3.5526 \times 10^{-2}$ & 3.7882 \\
\hline $128 \times 128$ & $8.2890 \times 10^{-3}$ & 4.2859 \\
\hline $256 \times 256$ & $1.8368 \times 10^{-3}$ & 4.5127 \\
\hline
\end{tabular}

Since the exact solution is not available, we perform the grid refinement analysis against the solution from the finest mesh, $M=N=512$ in Table 4.1, for the case $c^{-}=2$ and $c^{+}=1$. From the analysis described in [10], we know that the ratio in Table 4.1 approaches number 5 for a second order method, and number 3 for a first order method. The result in Table 4.1 shows nearly second order convergence of our method for the example.

As we increase the weight $c^{-}$using the same boundary condition as in Fig. 4.2 (a), there is more contribution to the energy from inside of the medium. As a result, the minimal surface will move up within the inside medium. If $c^{-}$is large enough, then the minimal surface will move up further until it is eventually barely touches the interface. However, once an intermediate solution does not touch the interface, it will not feel the difference of the mediums and the next iteration will move it towards the solution in the homogeneous medium which is a plane in our example. Because the plane passes the medium inside, it will move up again. For this case, the weighted minimal surface barely touches the medium inside, see Fig. 4.3 (b). To prevent the intermediate approximation from jumping back and forth, we solve the parabolic equation (3.7) so that the solution will not change too much at one iteration. In Fig. 4.3 (a), we show the result of an intermediate approximation to the solution while Fig. 4.3 (b) is 
the computed approximation which barely touches the interface. Note that, once the surface cuts through a small portion of the sphere, the curve, which is the intersection of the sphere and the surface, and its projection on the $x-y$ plane have large curvature. This may lead to numerical difficulties in resolving the curve and result in some errors of the computed solution to the parabolic equation which is visible from Fig. 4.3 (b). The size of error can be controlled by the parameter $\Delta t_{2}$. The smaller $\Delta t_{2}$ is, the smaller of such error. However, it requires more iterations if $\Delta t_{2}$ is small.

(a)

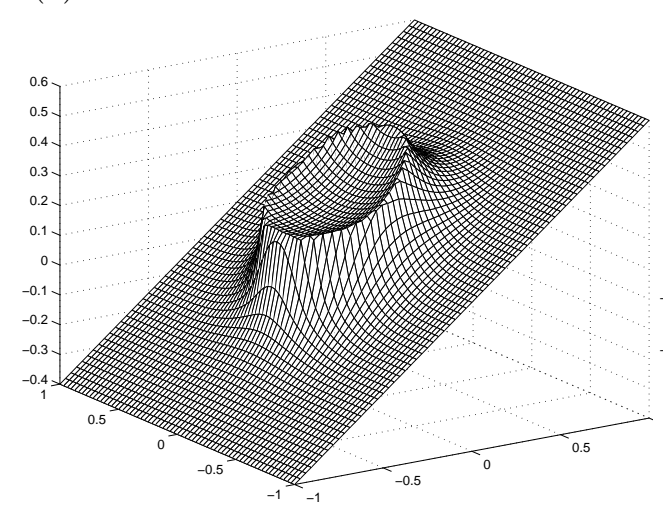

(b)

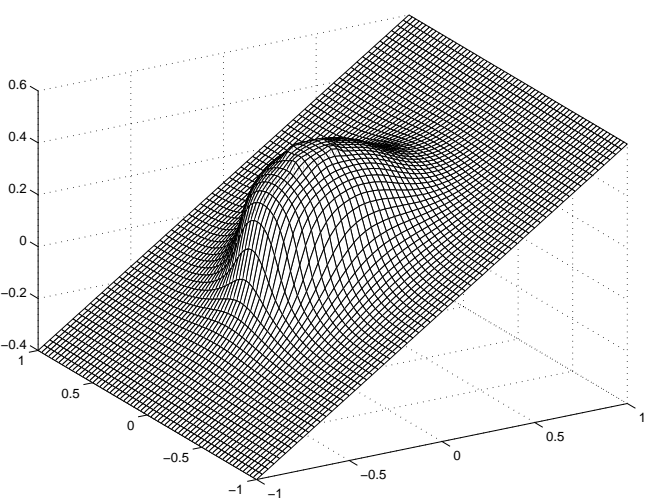

FIG. 4.3. Computed results with $c^{-}=20$ and $c^{+}=1$. The initial guess is $u^{0}(x, y)=-0.4+(x+$ 1)/2 (a) An intermediate solution in which the surface moves upwards to avoid large contribution from inside of the medium. (b) The accepted solution which is almost tangent of the sphere.

As a last test, we show the solution depends on the initial condition as well. The boundary condition is taken as a constant $\left.u(x, y)\right|_{\partial \Omega}=-0.65$. The weights are $c^{-}=1$ and $c^{+}=10$ so the minimal surface will try to avoid outside as much as possible. If the initial condition is taken as the same constant $\left.u^{0}(x, y)\right|_{\partial \Omega}=-0.65$, then it stays there because it is one of the local minimum and satisfies the Euler Lagrange equation plus the jump condition. However, it may not be the global minimum which is given in Fig. 4.4. In order to get the global minimum, the initial guess has to be carefully chosen. In Fig. 4.4, the initial guess is chosen as a piecewise linear function

$$
u^{0}(x, y)= \begin{cases}-0.65+\frac{0.65(x+1)}{0.4}, & \text { if }-1 \leq x \leq-0.6, \\ 0, & \text { if }-0.6<x \leq 0.6, \\ -0.65+\frac{0.65(x-1)}{0.4}, & \text { if } 0.6<x \leq 1 .\end{cases}
$$

5. Conclusion. In this paper, the weighted minimal surface problem involving two media has been studied both analytically and numerically. A generalized Snell's law for the weighted minimal surface at the interface between two different media is derived. A numerical method is developed to compute the weighted minimal surface which satisfies a non-linear elliptic equation with jump conditions at a free boundary, the intersection of the minimal surface and the interface between two media.

Acknowledgment. We would like to thank Dr. K. Ito of North Carolina State University for some useful suggestions and discussions. The first author is partially supported by ARO grants 39676-MA and 43751-MA and NFS grants DMS-0073403 


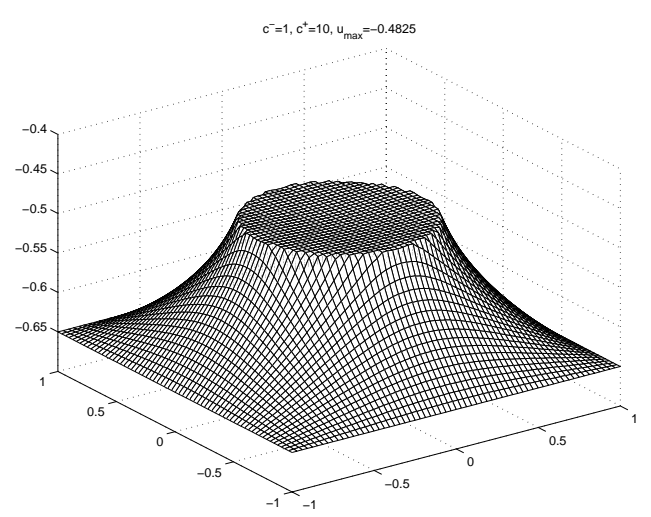

FIG. 4.4. Computed results with $c^{-}=1$ and $c^{+}=10$. The initial guess is given in (4.2). The initial condition is important to get the global minimum. Another obvious local minimum is the plane $u(x, y)=-0.65$.

and DMS-0201094. The second author is partially supported by an NSF grant DMS9973105. The last author is partially supported by an ONR grant N00014-02-1-0090, a DARPA grant N00014-12-1-0603, and the Sloan Foundation Fellowship.

\section{REFERENCES}

[1] L. M. ADAMS AND Z. LI, The immersed interface/multigrid methods for interface problems, SIAM J. Sci. Comput., 24 (2002), pp. 463-479.

[2] P Concus, R Finn, and D.A. Editors Hoffman, Geometric Analysis and Computer Graphics, Mathematical Sciences Research Institute Publications, Proceedings of a workshop held May 23-25, 1988.

[3] D. De ZeEuw, Matrix-dependent prolongations and restrictions in a blackbox multigrid solver, J. Comput. Appl. Math., 33 (1990), pp. 1-27.

[4] J. Douglas, Solution of the problem of plateau, Trans. Amer. Math. Soc., 33 (1931), pp. 263-321.

[5] E Dubois-Violette and B. Editors Pansu, International Workshop on Geometry and Interfaces, Aussois, France., September, 17-22, 1990.

[6] R Finn, Equilibrium Capillary Surfaces, Springer-Verlag, 1986.

[7] Enrico Giusti, Minimal surfaces and functions of bounded variation, Birkhäuser Verlag, Basel, 1984.

[8] Michael Grüter, Optimal regularity for codimension one minimal surfaces with a free boundary, Manuscripta Math., 58 (1987), pp. 295-343.

[9] K. Iто AND Z. LI, The immersed interface method for nonlinear interface problems with an application, submitted, 2002.

[10] Z. LI, The Immersed Interface Method - A Numerical Approach for Partial Differential Equations with Interfaces, $\mathrm{PhD}$ thesis, University of Washington, 1994.

[11] Z. LI, A fast iterative algorithm for elliptic interface problems, SIAM J. Numer. Anal., 35 (1998), pp. 230-254.

[12] Z. Li AND K. ITO, Maximum principle preserving schemes for interface problems with discontinuous coefficients, SIAM J. Sci. Comput., 23 (2001), pp. 1225-1242.

[13] T. Radó, On the Problem of Plateau, Ergeben. d. Math. u. ihrer Grenzgebiete, Berlin: SpringerVerlag, 1933.

[14] G ShAPIRO, The mathematics of soap films: explorations with Maple, American Mathematical Sociaty, 2000.

[15] G Shapiro, Geometric Partial Differential Equations and Image Analysis, Cambridge University Press, 2001.

[16] Leon Simon, Lectures on geometric measure theory, Australian National University Centre for Mathematical Analysis, Canberra, 1983. 
[17] Monica Torres, Plane-like minimal surfaces in periodic media with exclusions, Submmited to SIAM, Journal on Math. Analysis. 